\title{
A slow process in confined polymer melts: layer exchange dynamics at a polymer solid interface
}

\author{
L. Yelash, P. Virnau, and K. Binder \\ Institute of Physics, Johannes-Gutenberg-University, 55099 Mainz, Germany \\ W. Paul* \\ Institute of Physics, Martin-Luther-University, 06099 Halle, Germany
}

(Dated: March 31, 2022)

\begin{abstract}
Employing Molecular Dynamics simulations of a chemically realistic model of 1,4-polybutadiene between graphite walls we show that the mass exchange between layers close to the walls is a slow process already in the melt state. For the glass transition of confined polymers this process competes with the slowing down due to packing effects and intramolecular rotation barriers.

PACS numbers: 82.35.Gh,64.70.Q-,81.05.Qk
\end{abstract}

Polymer dynamics in confinement is both of fundamental interest concerning our understanding of the glass transition [1, 2], as well as of high technological importance for the performance of composite materials [3]. In experiments [4 -7] as well as simulations [1, 8-10] varying effects of confinement on the glass transition and even conflicting results have been observed for one and the same polymer-wall system [4 [6]. Obviously, the dynamics will be inhomogeneous as well as anisotropic in the vicinity of the confining surface, but it is not clear how large this effect is and how its extent into the bulk of the polymer is connected with structural anomalies close to the surface. These in turn will sensitively depend on the details of the polymer-solid interactions. Thus we found it highly desirable to perform a detailed simulation analysis of a well defined chemically realistic polymer-wall system and take advantage of the strength of the simulation approach to provide insight not (yet) obtainable from experiment. The segment length of the polymer and the radius of gyration of the chains have been identified as the relevant length scales over which the wall effect changes different aspects of the polymer dynamics [1], i.e., length scales from the sub-nanometer scale to at most $10 \mathrm{~nm}$. When one tries to model the mechanical properties of polymer composites on the other hand, one often has to assume a region of modified viscoelasticity within the polymer of several hundred nanometers extent [3]. The origin of the discrepancy between these two findings is not understood.

In bulk glass forming polymers one has a competition between two mechanisms leading to a time scale separation between vibrational and relaxational degrees of freedom, the caging effect as captured by mode coupling theory [11], which is essentially a hard sphere packing phenomenon, and the time scale separation introduced by the presence of rotational barriers in the chains $[12,13$. The latter mechanism is absent in the widely used coarse-

*Electronic address: Wolfgang.Paul@Physik.Uni-Halle.De grained models 1, 14, 15]. Both mechanisms compete upon approaching the glass transition in polymer melts. As we will show, attractive confining walls introduce another mechanism for time scale separation. This mechanism is the slow desorption kinetics of monomers at the surface (which in our case is graphite) leading to a slow layer exchange dynamics on the scale of (at least) the radius of gyration of the chains.

Simulation method We have performed Molecular Dynamics (MD) simulations of a well-validated, chemically realistic model [16 19] of a 1,4-polybutadiene (PBD) melt at a graphite surface. Unlike other studies using polyethylene [20, 21] , a polymer that easily crystallizes, we focus here on a glassforming polymer which has been extensively characterized in the bulk. The current model quantitatively reproduces all available experimental results on PB bulk dynamics [16 19] and its glass transition temperature of about $180 \mathrm{~K}$. The $(0,0,1)$ graphite surfaces are represented by layers positioned at $z=0, z=D=9.97 \mathrm{~nm}$, and $z=10.31 \mathrm{~nm}$ with a periodic boundary condition imposed at $L_{z}=10.65 \mathrm{~nm}$. All graphite carbon atoms are kept at fixed positions in the simulation. The force field for graphite is taken from [22] and Lorentz-Berthelot combining rules for the Lennard-Jones interactions are used. In the $x$ and $y$ directions periodic boundary conditions are employed at $L_{x}=14.91 \mathrm{~nm}$ and $L_{y}=14.76 \mathrm{~nm}$, respectively. The density at the center of the simulation box is adjusted close to its bulk value at the respective temperature, and the melt structure is equilibrated with a sequence of Langevin dynamics simulations at reduced excluded volume under $N V T$ conditions and MD post equilibration. Finally, we performed $N V T$ MD using the GROMACS package [23].

Results The dispersive interactions at the interface between both materials lead to a strong layering of the polymer segments at the graphite surface. Furthermore, for the chains of 29 repeat units which we have simulated, there is also an observable layering on the scale of the whole polymer chain due to the correlation hole effect 24]. In Fig. 1 the monomer density and the density of 
chain center of mass positions are shown for $T=353 \mathrm{~K}$ (about $2 T_{a}$ ), normalized to their bulk values.

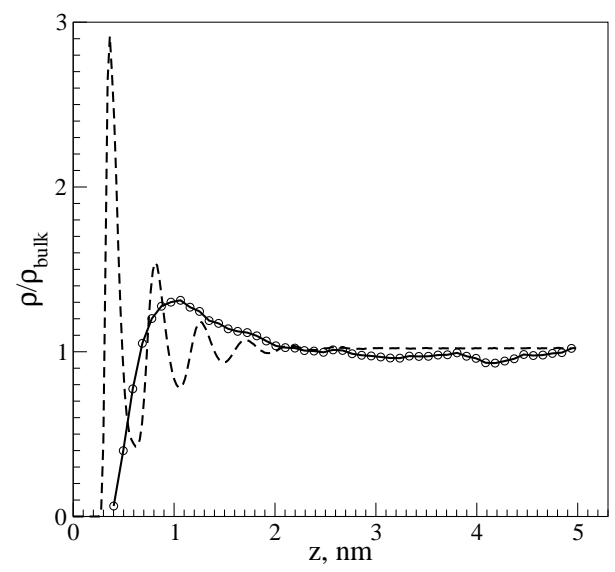

FIG. 1: Layering effects in the density (dashed line) and chain center of mass density (connected circles) of a confined polybutadiene melt. Both curves are normalized by the corresponding densities in the bulk.

We checked that the density dependence we obtained in the simulation was symmetric with respect to the distance to the two walls to judge the quality of equilibration. Fig. 1 displays half of the symmetrized profile. The length scale involved in the density layering is the size of the monomers (width of the chains), $\sigma \simeq 0.5 \mathrm{~nm}$. The perturbation of the melt structure propagates for about $2.5 \mathrm{~nm}$ from the graphite surface. Over the same distance, we observe a layering effect in the center of mass positions of the chains. The maximum in this density lies around $1 \mathrm{~nm}$ which is slightly smaller than the radius of gyration of the chains in the bulk because chains within this region of increased density are oriented parallel to the surface, i.e., their gyration tensor ellipsoid orients with its long axes in the $x, y$-plane. In contrast to the bulk behavior, at a wall the correlation hole effect therefore introduces a well defined liquid like layering in the center of mass density.

Both of these layering effects are connected with inhomogeneous dynamics in the film which we will trace to the slow adsorption/desorption process of monomers. Let us first focus on the orientational dynamics on the segmental scale as would be observable in nuclear magnetic resonance (NMR) experiments. We will study the following correlation function 25]

$$
C(t)=\left\langle P_{2}(\cos [\theta(t)]) P_{2}(\cos [\theta(0)])\right\rangle,
$$

where $\theta(t)$ is the angle between the orientation of the double bond in the polybutadiene monomer and a given magnetic field orientation, $P_{2}$ is the second Legendre polynomial and the angular brackets indicate a thermal average. We have determined this correlation function in a layer resolved manner with a total of 8 layers between the two

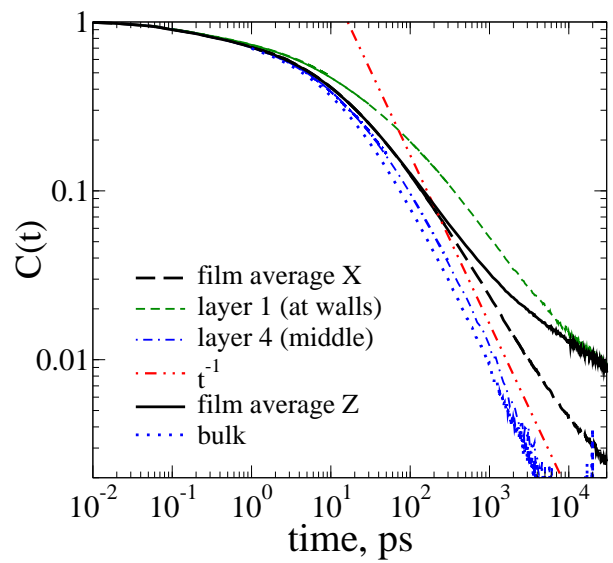

FIG. 2: Inhomogeneous rotational dynamics of bonds as showing up in an NMR correlation function $C(t)$ defined in the text. We show the relaxation function for two different layers in the film and the average relaxation in the film for an orientation of the external field parallel to the walls (X). We compare this to the bulk relaxation and the average film relaxation for an external field oriented perpendicular to the walls (Z).

graphite surfaces at $z=0$ and $z=D$. A given double bond is contributing to the relaxation function for layer $j$ if its center is within this layer at $t=0$. Most of the relaxation functions are shown for an orientation of an external magnetic field parallel to the graphite surface (x-direction). One can clearly observe that the dynamics is slowed down strongly, going from the center of the film (blue, dash-dotted line) to layer 1 (green, dashed line) directly at the walls. The relaxation in the center of the film is almost the same as in bulk (blue, dotted line) which was determined separately. The average signal from the thin film is shown by the black dashed curve for a field orientation parallel to the walls and by the black continuous curve for a field orientation perpendicular to the walls (z-direction). For the x-orientation the relaxation of the last percent of correlations is about an order of magnitude slower than in the bulk, whereas for the z-direction this relaxation appears to be slowed down by 2 to 3 orders of magnitude! For both relaxation functions a final slow process seems to be setting in around 2 ns.

In the bulk such a process (around $2 \%$ of the decay) in the $C-H$ bond reorientation was shown to be due to a coupling of local to large scale relaxation, i.e., conformational relaxation of the whole chain 26]. At the wall we are here observing a similar coupling which is furthermore most prominent for the perpendicular reorientation. To quantify the large scale motion of the chain in the vicinity of the wall we are presenting in Fig. 3 the mean square displacement of the center of mass of the chains distinguished with respect to directions parallel and normal to the walls. This time we have resolved 7 


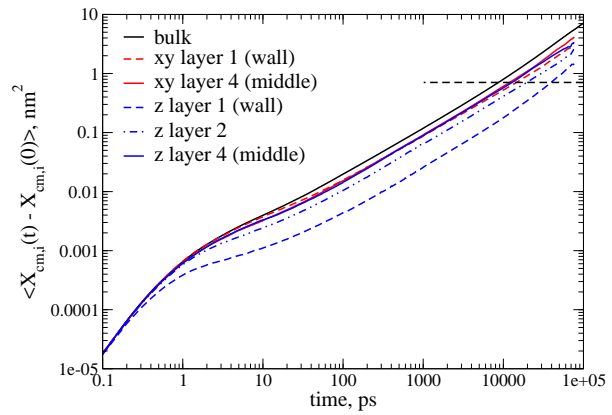

FIG. 3: Mean-square-displacement of the center of mass of chains for different layers chosen to have a thickness equal to the bulk $R_{g}$. We differentiate between the average displacement parallel to the walls, xy, and perpendicular to the walls, $\mathrm{z}$.

layers, i.e., every layer has a thickness of the order of the bulk $R_{g} \simeq 1.4 \mathrm{~nm}$. A chain contributes to the displacement function of layer $i$ when its center of mass is in that layer at $t=0$. In the bulk, all displacements in different Cartesian directions are equal and the same is true for the displacement we observe in the middle of the film (layer 4, blue and red continuous lines). However, due to a slightly larger density than for the bulk simulations, the displacement in the center of the film is still about $30 \%$ slower than in the bulk. We can quantify this by using an estimate of the Rouse time, defined by $\left\langle\left(\Delta X_{c m, i}\left(\tau_{R}\right)\right)^{2}\right\rangle=R_{g}^{2} / 3 \simeq 0.7 \mathrm{~nm}^{2}$. For the bulk this yields $\tau_{R}^{\text {bulk }} \simeq 9$ ns and for the center of the film we obtain $\tau_{R}^{\text {middle }} \simeq 12$ ns. At the wall, in layer 1 , the motion of the chain is not yet isotropic on a displacement scale of $R_{g}$. For the displacement parallel to the graphite surfaces we read off a Rouse time $\tau_{R}^{1, \mathrm{x}} \simeq 15 \mathrm{~ns}$, whereas for the displacement perpendicular to the walls it is $\tau_{R}^{1, \mathrm{z}} \simeq 39 \mathrm{~ns}$. It is interesting to note that from about 1 ps to about $50 \mathrm{ps}$ the displacement parallel to the graphite walls in layer 1 is actually slightly faster than the corresponding displacement in the center of the film. Torsional transitions, which are the relevant local motions on these time scales are, actually slowed down close to the wall (not shown), so we take this as an indication that dynamical correlations between local motions are changed in the vicinity of the wall.

One could be tempted to describe the difference between parallel and perpendicular mobility by assigning different friction constants to the motions in different directions, however, such an approach fails to account for the short time (1 to $10 \mathrm{ps}$ ) plateau of the perpendicular displacement. This plateau signifies a time scale separation between short time vibrational motion (up to $1 \mathrm{ps}$ ) and longer time relaxational motion.

In the bulk, caging due to packing effects and in- tramolecular barriers sets in around $T=273 \mathrm{~K}[12]$ and both effects can not be the reason for the plateau in the perpendicular displacement, as we have observed that the parallel motion close to the wall is as fast as the motion in the bulk. The new slow process occurring with polymers at attractive walls is the adsorption and desorption kinetics [27, 28] of monomers at the wall, and phenomenologically it gives rise to the same two-step relaxation behavior as the other two mechanisms.

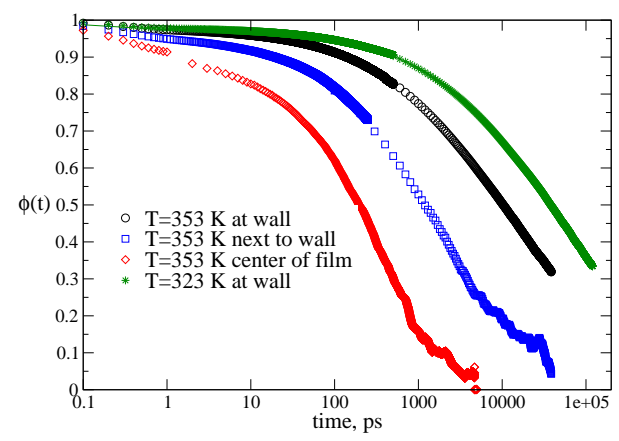

FIG. 4: Adsorption autocorrelation function for single monomers. The assignment to layers is done on the basis of the position of the center of mass of the chain at $t=0$, where by definition at $t=0$ the considered monomer has to be attached to the graphite surface.

We quantify the desorption kinetics by the following correlation function

$$
\phi(t)=\frac{\langle s(t) s(0)\rangle-\langle s\rangle^{2}}{\langle s(0) s(0)\rangle-\langle s\rangle^{2}}=\frac{\langle s(t)\rangle-\langle s\rangle^{2}}{1-\langle s\rangle^{2}},
$$

where $s(t)=1$ if the monomer is adsorbed (i.e., is within the first layer observable in the density profile) at time $t$ and $s(t)=0$ otherwise, and where we have employed the constraint $s(0)=1$ for the collection of the autocorrelation function. In Fig. 4 we can see that this autocorrelation function decays on a time scale comparable to the relaxation time defined from the mean squared center of mass displacement in z-direction (see Fig. 3). Furthermore, especially for the monomers at the wall, a clear two-step decay is observable with a plateau extending over the same time interval as the one observed for the center of mass msd and a plateau height above 0.95. We also observe that this plateau extends further at the lower temperature of $T=323 \mathrm{~K}$ which we included in this figure. Defining a time scale for the desorption kinetics by $\phi\left(\tau_{w}\right)=0.5$, we read off $\tau_{w}=10 \mathrm{~ns}$ at $353 \mathrm{~K}$ and $\tau_{w}=38 \mathrm{~ns}$ at $323 \mathrm{~K}$. Their ratio of 3.8 is larger than the ratio of 2.4 we found between the structural relaxation times in the center of the film for these two temperatures, which in turn agree with the corresponding bulk relaxation times [19] at the same density. Therefore, already from our simulations well above $T_{g}$ we obtain an indication that the presence of this additonal slow pro- 
cess may have a strong influence on the glass transition of this confined system.

Conclusions Confining 1,4-polybutadiene between graphite walls creates layering effects in the density as well as the center of mass density extending to about $2 R_{G}$ away from the walls. Both structural features influence the way in which different correlation functions depend on the distance to the walls as well as the way in which they become anisotropic. We have shown for a doublequantum NMR signal that this anisotropy as well as the slowing down induced by the walls become observable experimentally in the final few per-cent of the decay. The anisotropy can be very large and should be observable in experiments of strongly confined systems where the direction of the probing field with respect to the walls can be controlled. In other relaxation functions, e.g., scattering experiments, averaging over different directions of the momentum transfer and over the whole confined sample, might lead to only small residual observable effects, although the alteration of the dynamics in the vicinity of the wall can be rather large. Experimentally it is therefore highly desirable to develop techniques which can resolve relaxation behavior as a function of distance to the walls with a resolution on the nano-meter scale and to perform orientationally resolved experiments. Our sim- ulations should be a guide to judge what is needed in this context,and hence allow to clarify the existing controversies of interpretation. We have shown that the slow desorption kinetics constitutes a third mechanism for time scale separation in glass forming polymers in addition to packing effects and conformational barriers present in bulk samples. How the competition between these mechanisms develops upon approaching the glass transition in different samples is a very interesting question which deserves further experiment and simulation studies. One might speculate that the extend over which the walls influence the visoelastic response of a confined melt [3] increases with decreasing temperature and increasing chain length ( $R_{g}$ dependent layering). However, to address such a question in simulations, a mapping of our atomistic model to a coarse-grained model which can then be studied on larger length scales needs to be performed, which remains as a challenge for future work.

Acknowledgment: We acknowledge funding through the German Science Foundation through the focused funding program SPP 1369, sub-project C2. We are grateful to the Jülich Supercomputer Center for computer time on the JUGENE and JUROPA computers through project HMZ03, and to the European network of excellence SoftComp for computer time.
[1] J. Baschnagel and F. Varnik, J. Phys.: Condens. Matter 17, R851 (2005).

[2] M. Alcoutlabi and G. B. McKenna, J. Phys.: Condens. Matter 17, R461 (2005).

[3] S. G. Advani, Processing and Properties Nanocomposites (Worlds Scientific, Singapore, 2006); D. Gay and S. V. Hoa, Composite Materials, Design and Applications (CRC Press, Boca Raton, 2007)

[4] A. N. Raegen, M. V. Massa, J. A. Forrest and K. DalnokiVeress, Eur. Phys. J. E 27, 375 (2008).

[5] A. Serghei, H. Huth, C. Schick and F. Kremer, Macromolecules 41, 3636 (2008).

[6] S. Kim, S.A. Hewlett, C.B. Roth and J.M. Torkelson, Eur. Phys. J. E 30, 83 (2009).

[7] P. A. O'Connell and G. B. McKenna, Science 307, 1760 (2005).

[8] G.D. Smith, D. Bedrov and O. Borodin, Phys. Rev. Lett. 90, 226103 (2003)

[9] J. A. Torres, P. F. Nealey and J. J. de Pablo, Phys. Rev. Lett. 85, 3221 (2000)

[10] H. Eslami and F. Müller-Plathe, J. Phys. Chem. B 114, 387 (2010).

[11] W. Goetze, Complex Dynamics of Glass-Forming Liquids: A Mode-Coupling Theory, (Oxford University Press, Oxford, 2008).

[12] S. Krushev, W. Paul, Phys Rev E 67, 021806 (2003).

[13] M. Bernabei, A. J. Moreno and J. Colmenero, Phys. Rev. Lett. 101, 255701 (2008).

[14] T. Pakula, J. Chem. Phys. 95, 4675 (1991).
[15] J. Baschnagel, K. Binder, M. Milchev, in Polymer Surfaces, Interfaces and Thin Films, A. Karim and S. Kumar Eds., (World Scientific, Singapore, 2000).

[16] G. D. Smith and W. Paul, J. Phys. Chem. A 102, 12001208 (1998).

[17] G. D. Smith, W. Paul, M. Monkenbusch, and D. Richter, J. Chem. Phys. 114, 4285-4288 (2001).

[18] W. Paul, D. Bedrov and G. D. Smith, Phys. Rev. E 74, 021501, (2006).

[19] W. Paul and G. D. Smith, Rep. Prog. Phys. 67, 11171185 (2004).

[20] K. C. Daoulas, V. A. Harmandaris and V. G. Mavrantzas, Macromolecules 38, 5780 (2005). V. A. Harmandaris, K. C. Daoulas and V. G. Mavrantzas, Macromolecules 38, 5796 (2005).

[21] O. Alexiadis, V. Mavrantzas, R. Khare, J. Beckers and A. Baljon, Macromolecules, 41, 987 (2008).

[22] W. A. Steele, Surf. Sci. 36, 317 (1973).

[23] B. Hess et al. J. Chem. Theory Comput. 4, 435 (2008); http://www.gromacs.org

[24] P. G. De Gennes, Scaling Concepts in Polymer Physics, (Cornell University Press, Ithaca, 1979).

[25] K. Saalwächter, Prog. Nucl. Mag. Res. Sp. 51, 1 (2007).

[26] W. Paul, G. D. Smith and D. Y. Yoon, Macromolecules 30, 7772 (1997).

[27] K. A. Smith, M. Vladkov, and J.-L. Barrat, Macromolecules, 38, 571 (2005).

[28] M. Erber et al., Polymer 51, 129 (2010). 